\title{
El coste de la obligatoria rehabilitación energética de la vivienda colectiva: ¿un problema social?
}

\section{The cost of the mandatory energy refurbishment of the collective housing: a social problem?}

$\underline{\text { I. Lizundia }}^{(*)}$, L. Etxepare ${ }^{(*)}$, M. Sagarna ${ }^{(*)}$, E. J. Uranga ${ }^{(*)}$

\section{RESUMEN}

Los cambios normativos derivados de la aprobación de la Ley 8/2013 de Rehabilitación, Regeneración y Renovación Urbana van a suponer un antes y un después en los habituales modos de plantear los procesos de reforma de los bloques de vivienda colectiva. El artículo analiza las consecuencias de dichos cambios en el ámbito de la obligada rehabilitación energética de edificios y su repercusión económica y social. El análisis se fundamenta en el estudio comparado de treinta y dos edificios rehabilitados en los últimos años, confrontándolos con el hipotético coste económico que la aplicación de dichos cambios hubiera supuesto en sus respectivos procesos de ejecución. Los resultados obtenidos evidencian que para alcanzar los objetivos previstos, resulta fundamental potenciar la oferta de ayudas públicas, sugeridas en la ley pero no reflejadas en la práctica, en aras de la sostenibilidad del proceso y para que las mejores intenciones no deriven en un problema social.

Se justifica analíticamente la teoría escogiendo un ejemplo de la obra de Félix Candela, ejecutada por medio de cáscaras cilíndricas largas de cubierta, y elaborando el proceso de cálculo necesario propuesto.

Palabras clave: Rehabilitación; Eficiencia energética; Normativa; Impacto económico; Ayudas públicas.

\section{ABSTRACT}

Policy changes resulting from the adoption of the Law 8/2013 will mark a before and an after, regarding the usual ways to set out the refurbishment of the collective housing blocks. This article analyses the consequences of such changes in the field of the mandatory energy refurbishment of the residential buildings and its economical and social impact. The analysis is based on a comparative study of thirty two buildings refurbished in the last years, confronting the cost of their refurbishment with the hypothetical economic cost that such policy changes would have brought about. Results obtained show that the planned objectives will not be achieved unless an ambitious public aid policy is not turned on. Even if they are suggested in the Law, this aid is not reflected in the practice. Without them, the process will not be sustainable and the good initial intentions would turn into a social problem.

Keywords: Refurbishment; Energy efficiency; Regulations; Economic impact; Public aids.

(*) Universidad del País Vasco (UPV/EHU) (España).

Persona de contacto/Corresponding author: inigo.lizundia@ehu.es (I. Lizundia)

ORCID: http://orcid.org/oooo-ooo1-7336-1083 (I. Lizundia); http://orcid.org/oooo-00o2-9849-1244 (L. Etxepare); http://orcid.org/oooo-0001-7409-030X (M. Sagarna); http://orcid.org/oooo-0002-3416-5511 (E. J. Uranga)

Cómo citar este artículo/Citation: I. Lizundia; L. Etxepare; M. Sagarna; E. J. Uranga. (2018). El coste de la obligatoria rehabilitación energética de la vivienda colectiva: ċun problema social? Informes de la Construcción, 70(551): e269. https://doi.org/10.3989/ic.59856

Copyright: (C) 2018 CSIC. Este es un artículo de acceso abierto distribuido bajo los términos de la licencia de uso y distribución Creative Commons Reconocimiento 4.o Internacional (CC BY 4.0). 


\section{INTRODUCCIÓN}

Los representantes de todos los conjuntos residenciales entrevistados en el ámbito de un Proyecto de Investigación (1) llevado a cabo en la Universidad del País Vasco coinciden en señalar que la única razón que llevó a sus respectivas juntas de propietarios a adoptar la decisión de acometer la rehabilitación de las fachadas o de la cubierta de sus respectivos edificios fue la constatación de evidentes lesiones en forma de humedades, fisuras, desprendimientos, etc. Aunque coincidentes en la causa, el alcance de la intervención difirió en cada uno de los casos según diversos factores: tipología, gravedad y extensión de las patologías; existencia o no de un correcto asesoramiento por parte de algún Administrador de Fincas profesional o de algún técnico habituado a procesos de reforma similares; situación económica y social de la vecindad; posibilidad de plantear una intervención más ambiciosa con el fin de optimizar los siempre costosos medios auxiliares, etc. Así, mientras algunas comunidades optaron por una mínima reparación de los daños, otras apostaron por una rehabilitación integral incorporando, incluso, una mejora de la eficiencia energética de la envolvente exterior.

Las exigentes condiciones impuestas, entre otras, por la Ley 8/2013, de 26 de junio, de rehabilitación, regeneración y renovación urbanas (2), $\mathrm{L}_{3} \mathrm{R}$ en adelante, y la consiguiente modificación del DB-HE del Código Técnico de la Edificación (3) como consecuencia de la transposición a la legislación estatal de varias Directivas y otras disposiciones legales europeas relativas a la eficiencia energética en el marco del denominado objetivo 20-20-20 obligan a reconsiderar el planteamiento descrito de forma radical, reduciendo la capacidad de decisión de las comunidades de propietarios y, más concretamente, la actual arbitrariedad en cuanto al ámbito de actuación. Los diferentes planes estratégicos de rehabilitación, tanto de ámbito estatal como autonómico, o los estudios sobre el estado actual y las necesidades del parque residencial existente publicados en los últimos tiempos como el realizado a iniciativa del Grupo de Trabajo sobre Rehabilitación (GTR), Una visión-país para el sector de la edificación en España (4), coinciden en señalar la urgente necesidad de afrontar la rehabilitación energética de gran parte de nuestros edificios, de los cuales el 30,17\% fueron construidos antes de 1960, el $10,81 \%$ en la década de los sesenta y el 15,36\% entre 1970 y 1980 , con un porcentaje respecto al total de viviendas para cada uno de los tres periodos referidos del 20,04\%, 14,17\% y $19,88 \%$, respectivamente (5).

E inmediatamente, surge la pregunta: ¿̇a cuánto asciende el coste económico del cambio de planteamiento? La relación de estudios realizados en los últimos años analizando las consecuencias económicas y la hipotética amortización a medio y largo plazo de la rehabilitación energética de los edificios de vivienda colectiva es interminable siendo muchos los que, solo a título de ejemplo, merecerían ser citados (6)(7)(8). A menudo, las conclusiones no son coincidentes, algo lógico teniendo en cuenta lo complejo que resulta extraer resultados universales de un análisis en el que influyen múltiples factores, muchos de los cuales tienen que ver con la particular forma de habitar de cada individuo y en el que, además, se han de utilizar metodologías, herramientas de cálculo, seguimiento y control absolutamente heterogéneos con resultados poco comparables entre sí (9). Frente al análisis de lo que acontece en el periodo posterior a la rehabilitación en el que se centran los estudios citados, el presente artículo pretende aportar una reflexión respecto al momento inmediatamente previo en el que, independientemente de los posibles beneficios y retornos económicos futuros, las Comunidades de Propietarios se ven obligadas a afrontar el coste íntegro de las obras.

Dicha reflexión se fundamenta en el estudio realizado en torno a un conjunto de edificios de vivienda colectiva rehabilitados durante los últimos años en el ámbito geográfico de la Comunidad Autónoma del País Vasco (CAPV), con el objetivo de establecer un análisis comparado del coste de la obra realizada en su momento frente a la inversión necesaria a día de hoy para conseguir un resultado equivalente cumpliendo los requisitos de la normativa vigente.

\section{EL CAMBIO NORMATIVO}

En el extenso preámbulo de la $\mathrm{L}_{3} \mathrm{R}$ se establecen claramente sus objetivos principales. Ángela de la Cruz, jurista que intervino activamente en su redacción, profundiza, en un interesante artículo al efecto (10), en los mecanismos establecidos en la ley para la consecución de los objetivos planteados. Evidentemente, la necesaria regulación para el cumplimiento de los objetivos establecidos en el Paquete 20-20-20 "Energía y Cambio Climático” de la Unión Europea, que obliga a todos los países integrantes, está en el origen fundamental de la norma y constituye el desarrollo argumental de la parte que se refiere específicamente a la rehabilitación de edificios. La necesidad de intervenir sobre el parque de viviendas existentes para conseguir, con criterios de mayor eficiencia, un importante ahorro energético y luchar contra el cambio climático parece primordial. No obstante, resulta llamativo que en dicho preámbulo al mencionar otras razones para su aprobación se hable, en términos preferentes, de "oportunidades de crecimiento y de empleo en el sector de la construcción... tras el desplome de dicho sector en España, a consecuencia de la crisis", admitiendo más adelante que uno de los objetivos primordiales es "ofrecer un marco normativo idóneo para permitir la reconversión y reactivación del sector de la construcción, encontrando nuevos ámbitos de actuación, en concreto en la rehabilitación edificatoria..." y que "la regulación que contiene esta norma se enmarca en un contexto de crisis económica, cuya salida depende en gran medida -dado el peso del sector inmobiliario en dicha crisis-, de la recuperación y reactivación -de cara sobre todo al empleodel sector de la construcción”. Es decir, se admite desde un principio que no solo priman los aspectos medioambientales o de ahorro energético sino que existe una necesidad imperante de generar nuevos nichos de trabajo en un sector como el de la construcción que debe ir trasladando su tradicional y casi exclusiva dedicación a la obra nueva al mercado de la rehabilitación, objetivo aplaudido por organizaciones empresariales como la CEOE (11). Consideramos relevante remarcar esta apreciación para entender gran parte del desarrollo normativo de la ley y las consecuencias económicas y sociales que se derivan de la misma y que van a constituir parte fundamental del presente análisis.

Como ha quedado anticipado, la $\mathrm{L}_{3} \mathrm{R}$ supone un punto de inflexión en la forma de afrontar la rehabilitación futura del parque residencial. Además del propio articulado, cuenta con una veintena de disposiciones finales que modifican gran parte del contenido de otras normas fundamentales que afectan al mundo de la construcción como la Ley del Suelo, la Ley de Propiedad Horizontal, la Ley de Ordenación de la Edificación (LOE) o el Código Técnico de la Edificación (CTE). 
De las exigencias normativas más novedosas cabría destacar tres:

1. Todos los edificios de vivienda colectiva con una antigüedad superior a 50 años o los que pretendan acogerse a ayudas públicas para acometer obras de rehabilitación deberán contar con un Informe de Evaluación de los Edificios (IEE) que certifique su estado de conservación, el grado de accesibilidad y el nivel de eficiencia energética (Artículo 4 y Disposición Transitoria Primera de la $\mathrm{L}_{3} \mathrm{R}$ ). La parte fundamental de dicho informe es la que se refiere al estado de conservación, siendo la única que obliga a la propiedad a ejecutar obras de subsanación en el caso de las lesiones consideradas más graves estableciendo, además, un calendario de actuación obligatorio. Sirve, por lo tanto, como pistoletazo de salida de todo el posterior proceso de intervención.

Los análisis del grado de accesibilidad y del nivel de eficiencia energética se limitan a aportar información del estado del edificio en relación a dichos aspectos. Aunque su objetivo fundamental es el puramente estadístico, dirigido a la Administración para ampliar el banco de datos del parque residencial y servir de punto de partida a la regulación de futuras normas y actuaciones, su inclusión no puede desvincularse de los calendarios obligatorios que sí establecen otras normativas. En materia de accesibilidad, por ejemplo, el redactor del IEE deberá razonar si el edificio es susceptible de realizar ajustes razonables, concepto que, de forma literal, recoge el Real Decreto Legislativo 1/2013 (12) que, en su Disposición Adicional Tercera, obliga a todos los edificios existentes a ejecutar dichos ajustes antes de fin de 2017. Por su parte, la calificación energética del edificio incluida en el IEE viene siendo utilizada por determinados organismos oficiales para otorgar ayudas públicas y financiación en función de la mejora energética lograda en la obra de rehabilitación planteada.

2. La sección HE 1 del "Documento Básico HE Ahorro de Energía” del CTE será de aplicación en cualquier trabajo de reforma en edificio existente (Artículo 1 del HE 1 ).

3. El cambio respecto al anterior DB-HE es sustancial. Si antes solo era de aplicación en determinados casos, cualquier intervención deberá ahora tenerla en cuenta.

Se limita la demanda energética (aunque en diferente medida según el grado de intervención) en todo tipo de obras de reforma (Artículo 2.2.2 del HE 1).

Este artículo viene a regular las condiciones de la intervención que establecía el artículo 1 del HE anteriormente citado. En los casos de renovación de más del $25 \%$ de la envolvente térmica, deberá limitarse la demanda energética conjunta del edificio mientras que para el resto de casos se establece un valor máximo de la transmitancia térmica del cerramiento. En la inmensa mayoría de los casos, estas limitaciones obligan a la incorporación de aislamiento térmico en las partes afectadas. Así, las tradicionales renovaciones de fachada consistentes en una simple sustitución del revestimiento exterior, sin mejora térmica alguna, pasan a mejor vida.

Todo ello obliga a reconsiderar el modo en el que se han venido planteando los procesos de rehabilitación de cualquier edificio de vivienda colectiva hasta el momento. Sirva como ejemplo el caso de una comunidad de vecinos que decide acometer la reforma de las fachadas con el fin de reparar las lesiones existentes y renovar, de paso, el revestimiento exterior. Lógicamente, pretenden acogerse a las posibles ayudas económicas públicas disponibles para este tipo de obras. Pues bien, tras los cambios normativos, deberá proceder, inexorablemente, a la contratación previa de un técnico competente para la redacción de un IEE y de un posterior proyecto de rehabilitación, en el caso de que la intervención alterara la "configuración arquitectónica" del edificio (Artículo 2.2.b de la LOE)(13), algo que ocurre en la mayoría de este tipo de reformas. El proyecto no podrá limitarse a la simple sustitución del revestimiento de acabado sino que deberá reducir la demanda energética mediante su correcto aislamiento incorporando, además, todas aquellas partidas necesarias para subsanar los daños del conjunto del edificio calificados como moderados y/o altos en el IEE. Tras pasar por las fases de solicitud de presupuestos a diferentes empresas contratistas, la posterior adjudicación de la obra, la tramitación de la licencia de obra municipal o la petición de posibles subvenciones públicas, la comunidad deberá proceder a la contratación de la Dirección Facultativa de la obra (Artículos 12 y 13 de la LOE) compuesta por un arquitecto como Director de Obra y de un arquitecto técnico como Director de la Ejecución de la Obra (aunque determinados Ayuntamientos están admitiendo formulas simplificadas de esta doble figura), así como de un Coordinador de Seguridad y Salud. Tras la ejecución de los trabajos, se redactará la Documentación Final de Obra incluyendo el Certificado de Subsanación de las lesiones calificadas como urgentes en el IEE.

\section{LA FINANCIACIÓN PÚBLICA EN EL CONTEXTO EUROPEO}

Parece obvio que un cambio normativo que suponga un cambio tan radical a la hora de plantear la rehabilitación de miles de edificios, con consecuencias económicas indiscutibles para el conjunto de la sociedad, deba ir asociada, necesariamente, a una implicación firme y comprometida de las diferentes administraciones públicas a la hora de ayudar a sufragar su coste. Y, efectivamente, así queda reflejado en el propio Preámbulo de la $\mathrm{L}_{3} \mathrm{R}$ donde se dice que “...se asegura la colaboración y la cooperación económica de la Administración General del Estado...”. La propia Comisión Europea, en un Informe sobre el apoyo financiero para la mejora de la eficiencia energética de los edificios publicado en 2013 (14), coincidiendo con la aprobación de la $\mathrm{L}_{3} \mathrm{R}$, llega a la conclusión de que para alcanzar los objetivos "...es imprescindible mejorar el apoyo financiero...” siendo “...los Estados miembros los principales responsables de garantizar una mayor rentabilidad de las inversiones..." y recalcando la vital importancia de "...la estrecha cooperación entre las autoridades públicas, las entidades de financiación y el sector de la construcción”. Las principales debilidades señaladas en dicho Informe para alcanzar las metas propuestas son los altos costes iniciales, los largos periodos de recuperación de la inversión, el riesgo crediticio, la todavía escasa concienciación de los usuarios respecto a los beneficios futuros de la eficiencia energética y la disparidad de mecanismos de financiación existentes en los diferentes países.

Parte de las ayudas económicas aportadas por la Unión Europea a través del Fondo Europeo de Desarrollo Regional (FEDER) a los diferentes estados miembros va dirigida a la rehabilitación energética del parque de viviendas. Ayudas que son gestionadas y complementadas con las medidas propias de las diferentes administraciones públicas estatales y 
locales según lo establecido en sus respectivos programas y planes estratégicos. Tal y como concluía el citado Informe, la disparidad de criterios a la hora de planificar las ayudas públicas en cada país es enorme resultando muy complicado establecer un mínimo análisis comparativo entre las mismas. No cabe aquí resumirlas todas pero solo por citar algunos ejemplos, mientras Francia apuesta por préstamos a tasa cero y reducciones fiscales, Italia se centra exclusivamente en éstas últimas. Alemania, por su parte, ofrece préstamos a bajo interés y subvenciones a fondo perdido según los valores de eficiencia obtenidos y Portugal se decanta por establecer convenios con entidades financieras privadas para facilitar préstamos en condiciones más favorables a los disponibles en el mercado. El poco tiempo transcurrido desde su implementación hace que no existen todavía datos comparativos sobre la mayor o menor efectividad de las diferentes medidas.

En el caso del estado español, tanto la Administración General del Estado como el Gobierno Vasco han aprobado diferentes decretos y han creado organismos diversos para regular y gestionar las posibles ayudas públicas. La situación actual es la siguiente:

Ámbito estatal:

- IDAE (Instituto para la Diversificación y Ahorro de la Energía): Organismo adscrito al Ministerio de Energía, Turismo y Agenda Digital y gestor del principal programa de ayudas a la rehabilitación energética de edificios en el ámbito estatal (PAREER II) (15). Similar al modelo de ayudas alemán, oferta préstamos a bajo interés y subvenciones a fondo perdido o, lo que viene a ser lo mismo, entregas dinerarias sin contraprestación, tal y como quedan definidas en la propia convocatoria. Tras escasos cuatro meses desde su lanzamiento en enero de 2016, el primer programa PAREER-CRECE finalizó en mayo de 2016 al haberse superado ampliamente el presupuesto inicialmente previsto de 200.000.000 Euros. Año y medio más tarde, en diciembre de 2017, IDAE lanza la segunda convocatoria PAREER II que cuenta con un presupuesto total de 125.658.00o Euros, considerablemente menor al del anterior programa.

\section{Ámbito autonómico (CAPV):}

- Gobierno Vasco: Gestionadas por Etxebide (Servicio Vasco de Vivienda), ofrece ayudas directas de hasta 2.000 euros a la Comunidad de Vecinos para cualquier tipo de obra de reforma en elementos comunes, no vinculadas necesariamente a la eficiencia energética. Una cantidad a todas luces insuficiente para este tipo de obras que apenas llega para sufragar el IEE. Ofrece, además, ayudas individualizadas consistentes en desgravaciones fiscales y/o en préstamos y subvenciones de escasa cuantía, para las rentas más bajas.

- Gobierno Vasco: Programa 'RENOVE Rehabilitación' y otros programas de ayuda a la rehabilitación de edificios, cofinanciadas hasta en un $50 \%$ mediante el FEDER. Subvenciones a proyectos integrales que contemplen la mejora de las condiciones de accesibilidad, habitabilidad y eficiencia energética de edificios plurifamiliares anteriores a 1980 y cuyas exigentes condiciones hacen que sean escasos los edificios acogidos a este programa en los últimos años.
Ámbito municipal:

- Son muy pocos los ayuntamientos que cuentan con algún tipo de ayuda a la rehabilitación de edificios. La fórmula más empleada entre los que las ofertan es la de aplicar algún tipo de reducción en el coste de la tasa por Licencia Municipal de obras siendo una reducida minoría los que ofrecen subvenciones a fondo perdido a las comunidades.

Resulta evidente que la situación actual de las ayudas ofrecidas dista mucho del impulso económico anunciado en el Preámbulo de la $\mathrm{L}_{3} \mathrm{R}$. A la ya comentada escasez o incluso coyuntural ausencia de los fondos previstos se unen los siguientes problemas:

- Las cantidades económicas a percibir son, en su gran mayoría, muy reducidas en comparación al montante total de la obra.

- La concesión o no de la subvención y su posible cuantía no se conoce, en la mayoría de los casos, hasta la finalización de los trabajos no constituyendo, por lo tanto, un factor decisivo y fundamental a la hora de afrontar la decisión de acometer la obra. Los diferentes propietarios deben de calcular y sufragar las correspondientes derramas económicas sin contar con hipotéticas futuras ayudas.

- Los programas de ayuda dependen de los respectivos presupuestos estatales y/o autonómicos estando, por ello, sujetos a decisiones cambiantes en función de las prioridades y de las políticas a desarrollar por los diferentes gobernantes en cada momento. La asignación presupuestaria para las diferentes ayudas varía año tras año y no puede dar respuesta a todas las solicitudes recibidas.

El tema de la financiación, tanto pública como privada, resulta, por lo tanto, un factor crucial a la hora de valorar una ley tan importante como la $\mathrm{L}_{3} \mathrm{R}$. Y es evidente que se trata de su punto más débil, tal y como recoge Juan Rubio del Val, firme defensor de la misma en su globalidad, en el clarificador título del apartado 9 de un artículo suyo relativo al tema: $L a$ financiación no resuelta de estas actuaciones de rehabilitación edificatoria (16).

\section{METODOLOGÍA}

El Proyecto de Investigación ha sido llevado a cabo por investigadores del Dpto. de Arquitectura y del Dpto. de Economía Financiera de UPV/EHU (Universidad del País Vasco) y ha consistido en el análisis de 32 conjuntos residenciales situados en los tres territorios de la CAPV (Tabla 1).

\subsection{Selección de casos}

- Aunque se han incluido conjuntos residenciales de Bizkaia y Araba, el mayor número de casos corresponde a Gipuzkoa, al tratarse del territorio histórico que ha concentrado la principal actividad rehabilitadora de edificios colectivos. Su mayor peso no supone una distorsión de los resultados económicos obtenidos ya que, tras el análisis de las diferentes partidas de obra, se ha constatado que los costes de producción y de mano de obra del sector de la construcción son prácticamente coincidentes.

- El estudio abarca edificios construidos entre finales de los años 40 y los 90, prevaleciendo los erigidos entre las décadas sesenta y setenta, periodo en el que más se edificó. Se han abarcado diferentes entornos urbanísticos, econó- 
Tabla 1. Edificios analizados.

\begin{tabular}{|c|c|c|c|c|c|c|}
\hline $\begin{array}{l}\mathrm{N}^{\circ} \\
\text { caso }\end{array}$ & $\begin{array}{c}\text { No de } \\
\text { bloques }\end{array}$ & $\begin{array}{c}\text { Datos } \\
\text { compositivos (1) }\end{array}$ & $\begin{array}{c}\mathrm{N}^{\mathrm{o}} \mathrm{de} \\
\text { Propietarios (2) }\end{array}$ & $\begin{array}{c}\text { Año de } \\
\text { construcción }\end{array}$ & $\begin{array}{c}\text { Año de } \\
\text { rehabilitación }\end{array}$ & Elementos rehabilitados \\
\hline 1 & 1 & $\mathrm{~PB}+4$ & 8 & 1956 & 1989 & Fachadas \\
\hline 2 & 1 & $\mathrm{~PB}+6$ & 12 & 1964 & 1995 & Fachadas \\
\hline 3 & 3 & $\mathrm{PS}+\mathrm{PB}+9+\mathrm{BC}$ & 252 & 1979 & 2004 & Cubierta + Fachadas \\
\hline 4 & 3 & $\mathrm{~PB}+4$ & 24 & 1965 & 2004 & Fachadas \\
\hline 5 & 2 & $\mathrm{PS}+\mathrm{PB}+4$ & 25 & 1970 & 2004 & Fachadas \\
\hline 6 & 1 & $\mathrm{PS}+\mathrm{PB}+4$ & 8 & 1965 & 2004 & Cubierta + Fachadas \\
\hline 7 & 1 & $\mathrm{~PB}+5$ & 10 & 1954 & 2005 & Cubierta + Fachadas \\
\hline 8 & 1 & $\mathrm{~PB}+5$ & 10 & 1954 & 2005 & Fachadas \\
\hline 9 & 4 & $\mathrm{~PB}+4$ & 36 & 1968 & 2006 & Cubierta + Fachadas \\
\hline 10 & 1 & $\mathrm{~PB}+9+\mathrm{A}$ & 21 & 1958 & 2007 & Cubierta + Fachadas \\
\hline 11 & 4 & $\mathrm{PS}+\mathrm{PB}+4(+4)$ & 48 & 1971 & 2007 & Fachadas \\
\hline 12 & 1 & $\mathrm{~PB}+15$ & 62 & 1969 & 2007 & Fachadas \\
\hline 13 & 1 & $\mathrm{~PB}+15$ & 60 & 1970 & 2009 & Cubierta + Fachadas \\
\hline 14 & 3 & $\mathrm{PS}+\mathrm{PB}+3$ & 85 & 1985 & 2009 & Cubierta + Fachadas \\
\hline 12 & 1 & $\mathrm{~PB}+11$ & 44 & 1970 & 2009 & Fachadas \\
\hline 16 & 4 & $\mathrm{~PB}+8$ & 64 & 1970 & 2010 & Fachadas \\
\hline 17 & 1 & $\mathrm{~PB}+3$ & 10 & 1946 & 2010 & Cubierta + Fachadas \\
\hline 18 & 3 & $\mathrm{~PB}+3$ & 18 & 1963 & 2010 & Cubierta + Fachadas \\
\hline 19 & 1 & $\mathrm{PS}+\mathrm{PB}+4+\mathrm{A}$ & 8 & 1964 & 2011 & Fachadas \\
\hline 20 & 1 & $\mathrm{PS}+\mathrm{PB}+2$ & 3 & 1949 & 2011 & Cubierta + Fachadas \\
\hline 21 & 1 & $\mathrm{~PB}+8$ & 36 & 1965 & 2012 & Fachadas \\
\hline 22 & 1 & $\mathrm{PS}+\mathrm{PB}+3$ & 12 & 1995 & 2012 & Fachadas \\
\hline 23 & 1 & $\mathrm{PS}+\mathrm{PB}+4$ & 20 & 1975 & 2012 & Fachadas \\
\hline 24 & 1 & $\mathrm{~PB}+9+\mathrm{BC}$ & 40 & 1969 & 2012 & Cubierta + Fachadas \\
\hline 25 & 1 & $\mathrm{~PB}+4$ & 10 & 1949 & 2013 & Cubierta + Fachadas \\
\hline 26 & 1 & $\mathrm{PS}+\mathrm{PB}+4$ & 24 & 1980 & 2013 & Fachadas \\
\hline 27 & 1 & $\mathrm{~PB}+4$ & 8 & 1965 & 2013 & Fachadas \\
\hline 28 & 1 & $\mathrm{PS}+\mathrm{PB}+14+\mathrm{A}$ & 70 & 1974 & 2013 & Fachadas \\
\hline 29 & 1 & $\mathrm{~PB}+8$ & 18 & 1974 & 2014 & Fachadas \\
\hline 30 & 1 & $\mathrm{~PB}+2$ & 8 & 1956 & 2015 & Cubierta + Fachadas \\
\hline 31 & 1 & $\mathrm{PS}+\mathrm{PB}+7$ & 28 & 1975 & 2015 & Fachadas \\
\hline 32 & 2 & $\mathrm{PS}+\mathrm{PB}+4$ & 58 & 1969 & 2015 & Fachadas \\
\hline
\end{tabular}

(1) PS:Planta sótano PB: Planta baja BC: Planta bajo cubierta A: Ático

(2) Incluyendo viviendas y locales comerciales

En cumplimiento del compromiso de confidencialidad acordado con la Propiedad, no se incluye su localización.

micos y sociales así como tipologías edificatorias diversas, desde bloques de baja altura con apenas tres viviendas, hasta bloques en altura de más de setenta.

- Todos los edificios seleccionados han sido objeto de reforma en los últimos años. Aunque durante el trabajo de campo se han obtenido datos de las intervenciones realizadas tanto en las fachadas como en la cubierta y en la eliminación de barreras arquitectónicas, el análisis que se expone a continuación se limita exclusivamente a los trabajos llevados a cabo en las fachadas al tratarse del único elemento constructivo que aparece afectado de forma homogénea en todos los casos analizados lo que permite concluir con un estudio comparativo fiable.

\subsection{Estudio de casos}

La metodología establecida para el estudio de los diferentes casos se resume en los siguientes pasos:
- Elaboración de una Ficha Tipo individualizada en la que se incluyen: datos generales; datos compositivos; datos clasificatorios; cronología, autoría, características y coste de las obras realizadas; normativa afectada; propuesta y coste teórico para alcanzar los requisitos actuales; características de la Comunidad de Vecinos y aspectos relativos a la gestión de la obra.

- Estudio de la documentación de proyectos y obras, tanto originales como de las reformas sufridas a lo largo de su vida, obtenida en archivos municipales, estudios de arquitectura y empresas constructoras de la que se han extraído datos de las características de los cerramientos, cubierta, estructura e instalaciones, presupuesto, pliego de condiciones, subvenciones, ayudas públicas solicitadas y adjudicadas, etc.

- Análisis de las diferentes Normativas vigentes en cada momento.

- Toma de datos in situ de los edificios seleccionados: estado actual, reportaje fotográfico, identificación de las solucio- 
nes constructivas realmente ejecutadas tanto inicialmente como en las diferentes reformas llevadas a cabo, etc.

- Entrevistas personales con representantes de la Comunidad de Vecinos, Administradores de Fincas, propietarios particulares, encargados de empresas contratistas y técnicos facultativos.

- Cálculo de la propuesta de intervención teórica a aplicar en cada uno de los casos para alcanzar los requisitos exigidos por la nueva Normativa. Para ello, se ha considerado la solución constructiva que se hubiera tenido que utilizar a día de hoy para lograr un acabado similar al planteado en la reforma llevada a cabo años atrás en la rehabilitación de las fachadas del edificio.

- Análisis comparado de los costes resultantes entre la obra realmente ejecutada y la teórica, objetivo fundamental del proyecto de investigación y que ha permitido establecer, obtener, comparar y discutir diferentes resultados y datos sobre aspectos económicos, constructivos, sociales, etc.

\section{RESUMEN DE LOS DATOS OBTENIDOS}

Los resultados obtenidos en los 32 casos analizados se resumen en la Tabla 2. En las primeras columnas y bajo el encabezamiento "Obra ejecutada en rehabilitación anterior", se listan: el año de ejecución de la obra de reforma; el tipo de solución constructiva aplicada; la intervención o no de Dirección Facultativa; la inclusión o no de aislamiento y, en su caso, su espesor; el coste total final de la obra, incluyendo el IVA, la licencia municipal, los honorarios en el caso de que hubiera intervención técnica tanto en proyecto como en obra y otros gastos, actualizados todos ellos a día de hoy según el incremento del IPC a efectos de poder efectuar una comparación efectiva y real de los datos; el coste total por superficie de fachada; $y$, por último, el coste total por propietario, incluyendo viviendas y locales. No se ha incluido el dato de las ayudas públicas solicitadas y adjudicadas al ser poco relevante debido al escaso número de casos en los que se solicitó (solo en algunas de las obras realizadas a partir de 2013) y a la escasa cuantía de las cantidades recibidas en proporción al coste total de la obra.

En la segunda mitad de la tabla, en las columnas encabezadas por "Obra teórica para cumplir requisitos actuales", se incluyen los datos, en este caso teóricos, que se obtendrían en una hipotética obra de rehabilitación de fachada ejecutada a día de hoy, cumpliendo la normativa vigente y para conseguir un acabado similar al logrado en su momento.

Dada la diversidad de casos analizados, antes de proceder a su discusión y para una mejor comprensión de los diferentes valores reflejados en la Tabla 2, conviene realizar algunas consideraciones:

Por un lado, están las características tipológicas de los edificios originales, muy diferentes entre sí. Desde el punto de vista dimensional, se han analizado desde pequeños edificios con envolventes verticales de apenas $240 \mathrm{~m}^{2}$ hasta bloques con tipología de torre con fachadas de más de $4.000 \mathrm{~m}^{2}$. El dato del número de propietarios resulta también muy variable con edificios de tan solo tres propietarios hasta conjuntos residenciales de más de doscientos cincuenta. El número de fachadas afectadas es otro factor a tener en cuenta. Y por último, están los elementos que configuran la fachada (paños ciegos, vuelos, balcones, terrazas, relieves, carpinterías, etc) y sus características constructivas en función de la época en la que fueron erigidas. Todas estas particularidades repercu- ten de forma determinante tanto en la relación 'superficie de fachada $/ \mathrm{n}^{0}$ propietarios' como en la relación 'superficie de fachada /superficie envolvente'.

Por otro lado, el tipo de rehabilitación de fachada realizado en su momento difiere en cada uno de los casos en función de su estado real, las lesiones existentes, los elementos afectados, las soluciones constructivas aplicadas, etc. Las intervenciones más sencillas, que coinciden con las obras más antiguas, se limitan a un simple cambio del revestimiento de acabado, sin incorporación de aislamiento y, normalmente, sin existencia de proyecto técnico ni dirección de obra. Por su parte, en las obras más recientes, lo habitual es que los trabajos incluyan, además de los paños verticales, el saneado, la reforma o la sustitución del conjunto de elementos constructivos que configuran la fachada: el perímetro estructural, los solados, falsos techos y barandillas de balcones y, en casos puntuales, las carpinterías.

Y por último, la hipotética intervención que se plantea para la rehabilitación de fachadas de cada uno de los edificios resulta también muy diferente. Para cada caso, se ha supuesto una solución constructiva adaptada a la normativa actual cuyo acabado final sea equivalente o se asemeje lo más posible al acabado realizado en su momento. Para ello, se han utilizado las dos tipologías más comúnmente empleadas en la inmensa mayoría de edificios rehabilitados o en proceso de rehabilitación hoy en día. Así, para los edificios con un revestimiento continuo como acabado final exterior se han propuesto soluciones tipo SATE (Solución de Aislamiento Térmico por el Exterior) mientras que para los edificios con aplacados de fachada de materiales diversos se han estudiado soluciones de fachada ventilada con acabados similares a los ya realizados. En las actuaciones más recientes, anteriores al cambio normativo pero coincidentes en cuanto a criterios de planteamiento de ahorro energético, la propuesta teórica ha coincidido prácticamente con la ejecutada, incrementándose, en todo caso, el espesor del aislante incorporado.

La combinación de estos tres parámetros (edificio original, rehabilitación ejecutada y rehabilitación teórica) hace que los costes finales obtenidos en cada uno de los casos sean muy diferentes y difícilmente comparables entre sí. No obstante, hay que recordar que tampoco era éste el objetivo último del estudio, el de obtener un coste medio definitivo y universal, sino el de aproximarnos, para cada uno de los casos, a la inversión real que habría que realizar para cumplir con los requisitos normativos actuales y, a su vez y tras un análisis del conjunto, obtener una visión global del coste de la rehabilitación energética en nuestros días.

\section{DISCUSIÓN DE RESULTADOS}

El incremento de la inversión necesaria para llevar a cabo la hipotética obra de rehabilitación tras los cambios normativos respecto a la obra anteriormente ejecutada resulta muy significativo en todos los casos. En las Figuras 1 y 2 se recogen las diferencias de coste por propietario y por superficie de fachada, respectivamente, tanto en cantidades netas como en porcentaje.

El primer dato a resaltar es el importante aumento del coste respecto a aquellos casos en los que la obra ejecutada en su momento no incluía la incorporación de una capa aislante (distinguidos mediante una columna rayada en la tabla). Se 
Tabla 2. Cuadro resumen de los resultados obtenidos para los 32 casos analizados.

\begin{tabular}{|c|c|c|c|c|c|c|c|c|c|c|c|}
\hline \multirow{3}{*}{$\begin{array}{l}\mathrm{N}^{\mathrm{o}} \\
\text { caso }\end{array}$} & \multirow{3}{*}{$\begin{array}{c}\mathrm{N}^{0} \text { Prop. (1) } \\
\begin{array}{c}\text { Superficie } \\
\text { de fachada }\end{array}\end{array}$} & \multicolumn{5}{|c|}{ OBRA EJECUTADA EN REHABILITACIÓN ANTERIOR } & \multirow{2}{*}{\multicolumn{5}{|c|}{$\begin{array}{c}\text { OBRA TEÓRICA PARA CUMPLIR REQUISITOS ACTUALES } \\
\text { Solución constructiva a aplicar para un acabado similar al anterior }\end{array}$}} \\
\hline & & Año & \multicolumn{4}{|c|}{ Solución constructiva aplicada } & & & & & \\
\hline & & DF (2) & $\begin{array}{l}\text { Aisl. } \\
(3)\end{array}$ & $\begin{array}{c}\text { Coste total de } \\
\text { la obra (4) }\end{array}$ & $\begin{array}{l}\text { Coste total por } \\
\text { superficie }\end{array}$ & $\begin{array}{c}\text { Coste total por } \\
\text { Propietario }\end{array}$ & DF (2) & $\begin{array}{l}\text { Aisl. } \\
\text { (3) }\end{array}$ & $\begin{array}{c}\text { Coste total de } \\
\text { la obra (4) }\end{array}$ & $\begin{array}{l}\text { Coste total por } \\
\text { superficie }\end{array}$ & $\begin{array}{c}\text { Coste total por } \\
\text { Propietario }\end{array}$ \\
\hline \multirow{2}{*}{1} & 8 Pr. & 1989 & \multicolumn{4}{|c|}{ Revestimiento continuo monocapa } & \multicolumn{5}{|c|}{ Fachada SATE con mortero acrílico } \\
\hline & $360 \mathrm{~m}^{2}$ & No & No & $10.305 €$ & $28 € / \mathrm{m}^{2}$ & $1.288 € / \operatorname{Pr}$ & Sí & $80 \mathrm{~mm}$ & $43.920 €$ & $122 € / \mathrm{m}^{2}$ & $5.490 € / \mathrm{Pr}$ \\
\hline \multirow{2}{*}{2} & 12 Pr. & 1995 & \multicolumn{4}{|c|}{ Revestimiento continuo pintura plástica } & Fachada & TTE con $m$ & tero acrílico & & \\
\hline & $350 \mathrm{~m}^{2}$ & No & No & $27.977 €$ & $79 € / \mathrm{m}^{2}$ & $2.331 € / \operatorname{Pr}$ & Sí & $80 \mathrm{~mm}$ & $42.700 €$ & $122 € / \mathrm{m}^{2}$ & $3.558 € / \mathrm{Pr}$ \\
\hline & 252 Pr. & 2004 & Revestim & to continuo mort & acrílico & & Fachada & ITE con $m$ & tero acrílico & & \\
\hline 3 & $20.544 \mathrm{~m}^{2}$ & No & No & $912.331 €$ & $44 € / \mathrm{m}^{2}$ & $3.620 € / \mathrm{Pr}$ & Sí & $80 \mathrm{~mm}$ & $2.168 .008 €$ & $105 € / \mathrm{m}^{2}$ & $8.603 € / \mathrm{Pr}$ \\
\hline & 24 Pr. & 2004 & Revestim & to continuo mort & acrílico & & Fachada & TTE con $m$ & tero acrílico & & \\
\hline 4 & $1.568 \mathrm{~m}^{2}$ & No & No & $129.686 €$ & $82 € / \mathrm{m}^{2}$ & $5.403 € / \operatorname{Pr}$ & Sí & $80 \mathrm{~mm}$ & $263.722 €$ & $168 € / \mathrm{m}^{2}$ & $10.988 € / \mathrm{Pr}$ \\
\hline & 25 Pr. & 2004 & Aplacado & rámico & & & Hoja exte & or autopor & ante pasante de fál & ca de ladrillo caravi & \\
\hline 5 & $2.578 \mathrm{~m}^{2}$ & Sí & No & $595.527 €$ & $231 € / \mathrm{m}^{2}$ & $23.821 € / \mathrm{Pr}$ & Sí & $80 \mathrm{~mm}$ & $824.450 €$ & $319 € / \mathrm{m}^{2}$ & $32.978 € / \mathrm{Pr}$ \\
\hline 6 & 8 Pr. & 2004 & Aplacado & rámico & & & Fachada & ntilada ac: & ado cerámico & & \\
\hline 0 & $912 \mathrm{~m}^{2}$ & Sí & No & $199.094 €$ & $218 € / \mathrm{m}^{2}$ & $24.886 € / \mathrm{Pr}$ & Sí & $80 \mathrm{~mm}$ & $268.290 €$ & $294 € / \mathrm{m}^{2}$ & $33.536 € / \mathrm{Pr}$ \\
\hline & $10 \mathrm{Pr}$. & 2005 & Aplacado & rámico & & & Fachada & ntilada ac: & ado cerámico & & \\
\hline 7 & $672 \mathrm{~m}^{2}$ & Sí & No & $113.334 €$ & $168 € / \mathrm{m}^{2}$ & $11.333 € / \mathrm{Pr}$ & Sí & $80 \mathrm{~mm}$ & $160.915 €$ & $239 € / \mathrm{m}^{2}$ & $16.091 € / \mathrm{Pr}$ \\
\hline 8 & $10 \mathrm{Pr}$. & 2005 & Aplacado & rámico & & & Fachada & ntilada ac: & ado cerámico & & \\
\hline 8 & $580 \mathrm{~m}^{2}$ & Sí & No & $82.258 €$ & $141 € / \mathrm{m}^{2}$ & $8.225 € / \mathrm{Pr}$ & Sí & $80 \mathrm{~mm}$ & $128.858 €$ & $222 € / \mathrm{m}^{2}$ & $12.885 € / \operatorname{Pr}$ \\
\hline & 36 Pr. & 2006 & Aplacado & rámico & & & Fachada & ntilada ac: & ado cerámico & & \\
\hline 9 & $2.050 \mathrm{~m}^{2}$ & Sí & No & $502.229 €$ & $244 € / \mathrm{m}^{2}$ & $13.950 € / \mathrm{Pr}$ & Sí & $80 \mathrm{~mm}$ & $596.550 €$ & $291 € / \mathrm{m}^{2}$ & $16.570 € / \mathrm{Pr}$ \\
\hline & 21 Pr. & 2007 & Revestim & to continuo mon & & & Fachada & TTE con $m$ & rtero acrílico & & \\
\hline 10 & $2.004 \mathrm{~m}^{2}$ & Sí & No & $384.161 €$ & $191 € / \mathrm{m}^{2}$ & $18.293 € / \mathrm{Pr}$ & Sí & $80 \mathrm{~mm}$ & $472.944 €$ & $236 € / \mathrm{m}^{2}$ & $22.521 € / \mathrm{Pr}$ \\
\hline 11 & $48 \mathrm{Pr}$. & 2007 & Hoja exte & r de fábrica conve & onal de ladrillo car & & Hoja exte & or autopor & ante pasante de fál & ca de ladrillo caravi & \\
\hline 11 & $3.728 \mathrm{~m}^{2}$ & Sí & $40 \mathrm{~mm}$ & $1.036 .122 €$ & $277 € / \mathrm{m}^{2}$ & $21.585 € / \mathrm{Pr}$ & Sí & $80 \mathrm{~mm}$ & $1.181 .300 €$ & $316 € / \mathrm{m}^{2}$ & $24.610 € / \operatorname{Pr}$ \\
\hline & $62 \mathrm{Pr}$. & 2007 & Aplacado & $\mathrm{n}$ aislante $+\mathrm{acab}$ & pétreo & & Fachada & ntilada ac: & ado pétreo & & \\
\hline 12 & $2.483 \mathrm{~m}^{2}$ & Sí & $60 \mathrm{~mm}$ & $507.059 €$ & $204 € / \mathrm{m}^{2}$ & $8.178 € / \mathrm{Pr}$ & Sí & $80 \mathrm{~mm}$ & $690.274 €$ & $278 € / \mathrm{m}^{2}$ & $11.133 € / \operatorname{Pr}$ \\
\hline & $60 \mathrm{Pr}$. & 2009 & Fachada & tilada acabado co & & & Fachada & ntilada ac: & ado continuo & & \\
\hline 13 & $3.045 \mathrm{~m}^{2}$ & Sí & $45 \mathrm{~mm}$ & $763.290 €$ & $250 € / \mathrm{m}^{2}$ & $12.721 € / \mathrm{Pr}$ & Sí & $80 \mathrm{~mm}$ & $781.345 €$ & $256 € / \mathrm{m}^{2}$ & $13.022 € / \operatorname{Pr}$ \\
\hline & 85 Pr. & 2009 & Aplacado & rámico & & & Fachada & ntilada ac: & ado cerámico & & \\
\hline 14 & $3.900 \mathrm{~m}^{2}$ & Sí & No & $1.196 .320 €$ & $306 € / \mathrm{m}^{2}$ & $14.074 € / \mathrm{Pr}$ & Sí & $80 \mathrm{~mm}$ & $1.218 .320 €$ & $312 € / \mathrm{m}^{2}$ & $14.333 € / \mathrm{Pr}$ \\
\hline & 44 Pr. & 2009 & Fachada & tilada acabado co & & & Fachada & ntilada ac: & ado continuo & & \\
\hline 15 & $2.851 \mathrm{~m}^{2}$ & Sí & $60 \mathrm{~mm}$ & $797.080 €$ & $279 € / \mathrm{m}^{2}$ & $18.115 € / \mathrm{Pr}$ & Sí & $80 \mathrm{~mm}$ & $819.654 €$ & $287 € / \mathrm{m}^{2}$ & $18.628 € / \mathrm{Pr}$ \\
\hline 16 & 64 Pr. & 2010 & Fachada & tilada acabado ce & & & Fachada & ntilada ac: & ado cerámico & & \\
\hline 10 & $4.350 \mathrm{~m}^{2}$ & Sí & $60 \mathrm{~mm}$ & $960.239 €$ & $220 € / \mathrm{m}^{2}$ & $15.003 € / \operatorname{Pr}$ & Sí & $80 \mathrm{~mm}$ & $985.395 €$ & $226 € / \mathrm{m}^{2}$ & $15.396 € / \mathrm{Pr}$ \\
\hline & $10 \mathrm{Pr}$. & 2010 & Fachada & TE con mortero a & & & Fachada & TTE con $m$ & tero acrílico & & \\
\hline 17 & $649 \mathrm{~m}^{2}$ & Sí & $60 \mathrm{~mm}$ & $142.887 €$ & $220 € / \mathrm{m}^{2}$ & $14.288 € / \mathrm{Pr}$ & Sí & $80 \mathrm{~mm}$ & $151.756 €$ & $233 € / \mathrm{m}^{2}$ & $15.175 € / \mathrm{Pr}$ \\
\hline 18 & $18 \mathrm{Pr}$. & 2010 & Aplacado & rámico & & & Fachada & ntilada ac: & ado cerámico & & \\
\hline 18 & $1.850 \mathrm{~m}^{2}$ & Sí & No & $387.734 €$ & $209 € / \mathrm{m}^{2}$ & $21.540 € / \mathrm{Pr}$ & Sí & $80 \mathrm{~mm}$ & $445.850 €$ & $241 € / \mathrm{m}^{2}$ & $24.769 € / \mathrm{Pr}$ \\
\hline 10 & 8 Pr. & 2011 & Fachada & tilada acabado co & osite aluminio & & Fachada & ntilada ac: & ado composite al & & \\
\hline 19 & $840 \mathrm{~m}^{2}$ & Sí & $40 \mathrm{~mm}$ & $254.700 €$ & $303 € / \mathrm{m}^{2}$ & $31.837 € / \mathrm{Pr}$ & Sí & $80 \mathrm{~mm}$ & $269.287 €$ & $320 € / \mathrm{m}^{2}$ & $33.660 € / \mathrm{Pr}$ \\
\hline P & 3 Pr. & 2011 & Fachada & TE con mortero a & & & Fachada & TTE con $m$ & tero acrílico & & \\
\hline 20 & $241 \mathrm{~m}^{2}$ & Sí & $40 \mathrm{~mm}$ & $79.566 €$ & $330 € / \mathrm{m}^{2}$ & $26.522 € / \operatorname{Pr}$ & Sí & $80 \mathrm{~mm}$ & $83.110 €$ & $344 € / \mathrm{m}^{2}$ & $27.703 € / \operatorname{Pr}$ \\
\hline 21 & 36 Pr. & 2012 & Fachada & tilada acabado ce & & & Fachad & ntilada ac: & ado cerámico & & \\
\hline 21 & $1.993 \mathrm{~m}^{2}$ & Sí & $80 \mathrm{~mm}$ & $490.278 €$ & $246 € / \mathrm{m}^{2}$ & $13.618 € / \mathrm{Pr}$ & Sí & $80 \mathrm{~mm}$ & $490.278 €$ & $246 € / \mathrm{m}^{2}$ & $13.618 € / \mathrm{Pr}$ \\
\hline 0 & $12 \mathrm{Pr}$. & 2012 & Fachada & TE con mortero a & & & & & Fachada SATE c & mortero acrílico & \\
\hline 22 & $1.210 \mathrm{~m}^{2}$ & Sí & $40 \mathrm{~mm}$ & $158.510 €$ & $131 € / \mathrm{m}^{2}$ & $13.209 € / \operatorname{Pr}$ & Sí & $80 \mathrm{~mm}$ & $171.820 €$ & $142 € / \mathrm{m}^{2}$ & $14.318 € / \mathrm{Pr}$ \\
\hline & $20 \mathrm{Pr}$. & 2012 & Fachada & tilada acabado fe & & & Fachada & ntilada ac: & ado fenólico & & \\
\hline 23 & $2.400 \mathrm{~m}^{2}$ & Sí & $50 \mathrm{~mm}$ & $623.525 €$ & $259 € / \mathrm{m}^{2}$ & $31.176 € / \mathrm{Pr}$ & Sí & $80 \mathrm{~mm}$ & $634.900 €$ & $264 € / \mathrm{m}^{2}$ & $31.745 € / \mathrm{Pr}$ \\
\hline 2 & 40 Pr. & 2012 & Fachada & TE con mortero a & & & Fachada & TTE con $m$ & tero acrílico & & \\
\hline 24 & $2.786 \mathrm{~m}^{2}$ & Sí & $60 \mathrm{~mm}$ & $421.590 €$ & $151 € / \mathrm{m}^{2}$ & $10.539 € / \mathrm{Pr}$ & Sí & $80 \mathrm{~mm}$ & $435.200 €$ & $156 € / \mathrm{m}^{2}$ & $10.880 € / \operatorname{Pr}$ \\
\hline 2 & $10 \mathrm{Pr}$. & 2013 & Fachada & TE con mortero a & & & Fachada & TTE con $m$ & tero acrílico & & \\
\hline 25 & $441 \mathrm{~m}^{2}$ & Sí & $60 \mathrm{~mm}$ & $104.489 €$ & $236 € / \mathrm{m}^{2}$ & $10.448 € / \mathrm{Pr}$ & Sí & $80 \mathrm{~mm}$ & $114.923 €$ & $260 € / \mathrm{m}^{2}$ & $11.492 € / \operatorname{Pr}$ \\
\hline 36 & 24 Pr. & 2013 & Rejuntad & erámico & & & Fachada & ntilada ac: & do cerámico & & \\
\hline 20 & $900 \mathrm{~m}^{2}$ & Sí & No & $129.982 €$ & $144 € / \mathrm{m}^{2}$ & $5.415 € / \mathrm{Pr}$ & Sí & $80 \mathrm{~mm}$ & $229.690 €$ & $255 € / \mathrm{m}^{2}$ & $9.570 € / \mathrm{Pr}$ \\
\hline 27 & 8 Pr. & 2013 & Fachada & tilada acabado ce & & & Fachada & ntilada ac: & ado cerámico & & \\
\hline 27 & $850 \mathrm{~m}^{2}$ & Sí & $50 \mathrm{~mm}$ & $173.355 €$ & $204 € / \mathrm{m}^{2}$ & $21.669 € / \operatorname{Pr}$ & Sí & $80 \mathrm{~mm}$ & $182.480 €$ & $214 € / \mathrm{m}^{2}$ & $22.810 € / \mathrm{Pr}$ \\
\hline 28 & 70 Pr. & 2013 & Fachada & tilada acabado co & osite aluminio & & Fachada & ntilada ac: & ado composite a & & \\
\hline 28 & $3.476 \mathrm{~m}^{2}$ & Sí & $60 \mathrm{~mm}$ & $842.365 €$ & $242 € / \mathrm{m}^{2}$ & $12.033 € / \mathrm{Pr}$ & Sí & $80 \mathrm{~mm}$ & $861.301 €$ & $276 € / \mathrm{m}^{2}$ & $12.304 € / \operatorname{Pr}$ \\
\hline ק & 18 Pr. & 2014 & Fachada & TE con mortero a & & & Fachada & TTE con $m$ & tero acrílico & & \\
\hline 29 & $1.150 \mathrm{~m}^{2}$ & Sí & $60 \mathrm{~mm}$ & $223.560 €$ & $194 € / \mathrm{m}^{2}$ & $12.420 € / \mathrm{Pr}$ & Sí & $80 \mathrm{~mm}$ & $234.470 €$ & $203 € / \mathrm{m}^{2}$ & $13.026 € / \mathrm{Pr}$ \\
\hline & 8 Pr. & 2015 & Fachada & TE con mortero a & & & Fachada & TTE con $m$ & tero acrílico & & \\
\hline 30 & $504 \mathrm{~m}^{2}$ & Sí & $80 \mathrm{~mm}$ & $101.648 €$ & $201 € / \mathrm{m}^{2}$ & $12.706 € / \mathrm{Pr}$ & Sí & $80 \mathrm{~mm}$ & $101.648 €$ & $201 € / \mathrm{m}^{2}$ & $12.706 € / \mathrm{Pr}$ \\
\hline & $28 \mathrm{Pr}$. & 2015 & Fachada & tilada acabado co & osite aluminio & & Fachada & ntilada ac: & ado composite a & & \\
\hline 31 & $1.100 \mathrm{~m}^{2}$ & Sí & $80 \mathrm{~mm}$ & $361.853 €$ & $328 € / \mathrm{m}^{2}$ & $12.923 € / \mathrm{Pr}$ & Sí & $80 \mathrm{~mm}$ & $361.853 €$ & $328 € / \mathrm{m}^{2}$ & $12.923 € / \operatorname{Pr}$ \\
\hline & 58 Pr. & 2015 & Fachada & tilada acabado co & osite aluminio & & Fachada & ntilada ac: & ado composite al & & \\
\hline 32 & $3.670 \mathrm{~m}^{2}$ & Sí & $80 \mathrm{~mm}$ & $989.478 €$ & $269 € / \mathrm{m}^{2}$ & $17.059 € / \mathrm{Pr}$ & Sí & $80 \mathrm{~mm}$ & $989.478 €$ & $269 € / \mathrm{m}^{2}$ & $17.059 € / \mathrm{Pr}$ \\
\hline
\end{tabular}




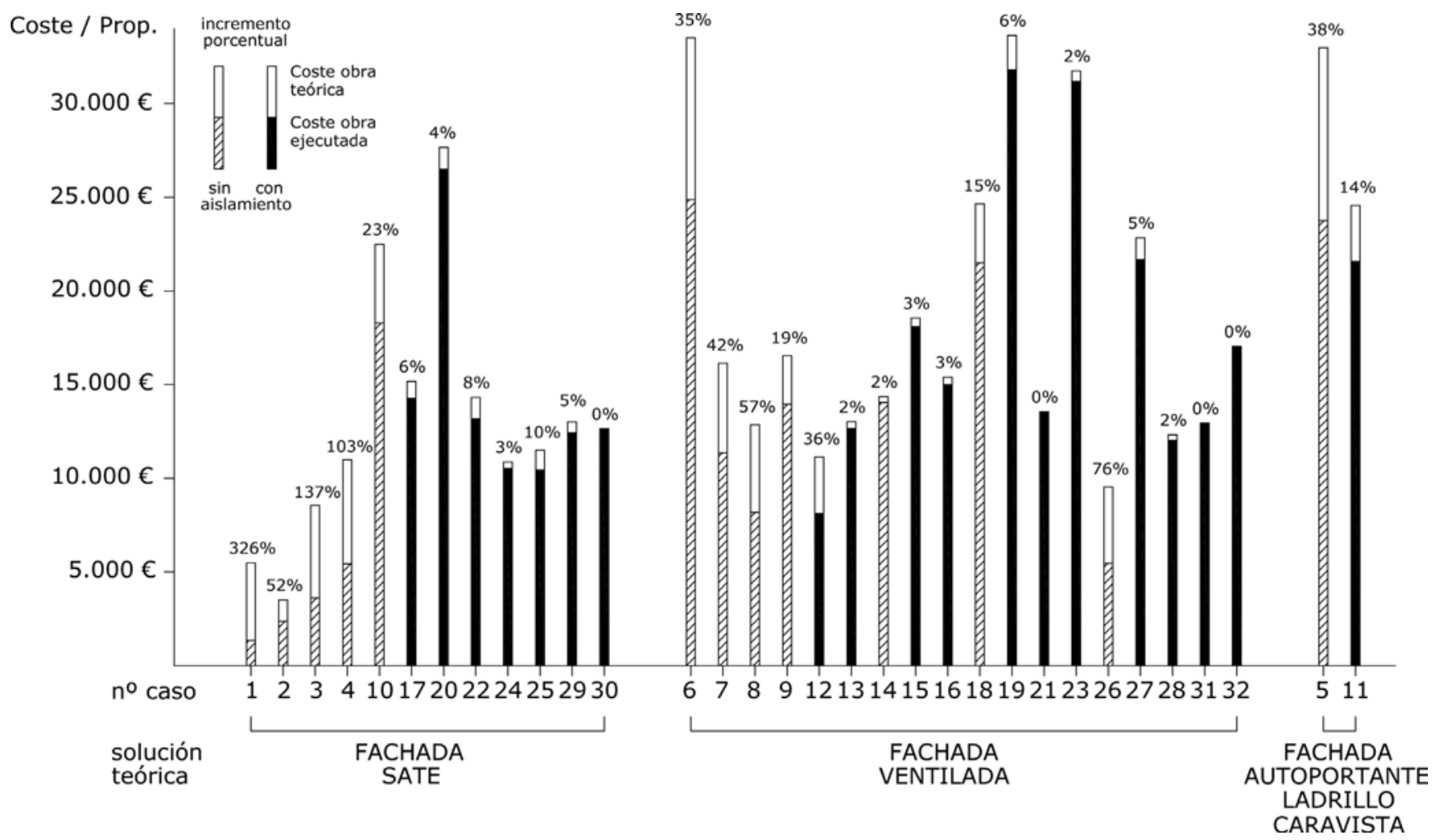

Figura 1. Diferencias de coste por propietario según el tipo de solución constructiva.

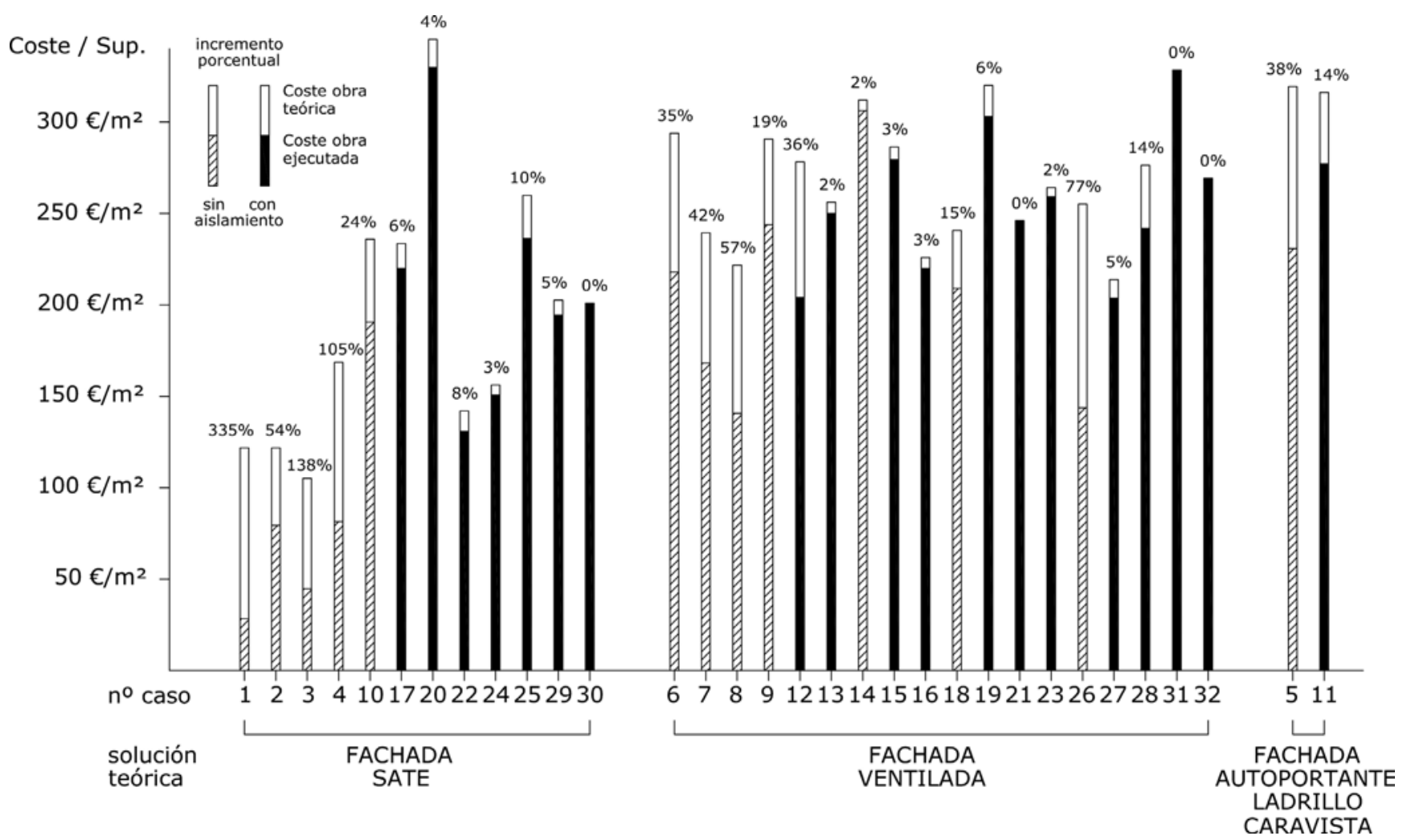

Figura 2. Diferencias de coste por superficie de fachada según el tipo de solución constructiva.

trata de las obras más antiguas limitadas a una simple mejora del acabado exterior. En el caso de las fachadas rematadas con un revestimiento continuo (pinturas, monocapas, etc, sobre un enfoscado previo), el incremento del coste medio respecto a una hipotética solución tipo SATE con un acabado similar incluyendo aislamiento supera el 60\%. Del mis- mo modo, en el caso de las fachadas rehabilitadas mediante aplacados cerámicos, pétreos, etc, sin mejora térmica alguna, el incremento de la inversión respecto a una fachada aislada cumpliendo las exigencias del nuevo DB-HE del CTE, alcanza una media del $30 \%$. En el caso de la única obra de rehabilitación de una fachada de fábrica de ladrillo caravista ejecutada 
sin aislamiento en la que se plantea una teórica reforma consistente en la eliminación de la hoja exterior y la incorporación de una nueva fábrica de ladrillo caravista autoportante y pasante, generando una cámara de aire aislada, el incremento de coste ascendería a un $38 \%$.

Las diferencias se reducen sustancialmente en aquellos casos en los que la obra ejecutada ya incorporaba una mejora térmica de la envolvente. Se trata de la mayor parte de las obras ejecutadas a partir de 2007 pese a no estar obligadas a ello por no exigirlo el DB-HE vigente entre 2006 y 2013, salvo los tres proyectos redactados a partir de finales de 2013 (casos 30-31-32) que sí se vieron afectados por el nuevo DB-HE. En todos los casos e independientemente de la normativa vigente en cada momento, el incremento porcentual del coste es muy similar, en torno a un $5 \%$ (salvo el caso 11 de fábrica de ladrillo visto que se eleva al $14 \%)$.

Como ya se ha comentado, la singularidad de cada caso hace que del estudio realizado no se puedan extrapolar cifras, ni siquiera aproximadas, del coste medio de la rehabilitación de una fachada en nuestros días. Los costes reales variarán significativamente en cada caso en función de múltiples variables. Además del tipo de solución constructiva aplicado a los paños generales, la posible intervención en otros elementos constructivos influye de forma muy significativa en el coste final. Desde el punto de vista económico y para la misma superficie de fachada, no será lo mismo limitarse a dotar de una nueva piel térmica a la envolvente exterior que incluir, además, el saneado completo del perímetro estructural y de los balcones. La relación coste/superficie variará considerablemente en uno u otro caso.

No obstante, sí se pueden avanzar algunos datos interesantes. Como era previsible, los costes resultantes en los casos en los que se opta por una solución de fachada ventilada mediante elementos prefabricados con una importante carga tecnológica son superiores respecto a los revestimientos continuos tipo SATE. El coste medio de los edificios estudiados con fachadas ventiladas de acabados diversos, incluyendo todos los conceptos (medios auxiliares, licencia, honorarios técnicos, IVA, etc), asciende a $267 € / \mathrm{m}^{2}$ mientras que en el caso de las fachadas tipo SATE, el coste medio por superficie se queda en $191 € / \mathrm{m}^{2}$. En las fachadas autoportantes de fábrica de ladrillo caravista el coste supera los $315 € / \mathrm{m}^{2}$.

Aunque conviene insistir en que estas cifras se limitan a los edificios analizados y no pueden universalizarse de forma genérica, sí que sirven para tener una idea del coste de la rehabilitación energética en nuestros días y su repercusión en la economía particular de cada uno de los propietarios de un edificio de vivienda colectiva. El coste de una intervención completa en las fachadas de un edificio consistente en soluciones tipo SATE puede superar fácilmente los $10.000 €$ por propietario (10.922 €/Pr. de media en los edificios analizados). En el caso de las fachadas ventiladas, el coste prácticamente se duplica, aproximándose a los $20.000 €$ por propietario (18.336 €/Pr. de media). Si, como ocurre en muchos casos, la obra de fachada va aparejada a una intervención en la cubierta del edificio (40\% de los casos objeto de estudio según se refleja en la Tabla 1), el coste de la inversión a realizar por la comunidad de propietarios se puede disparar por encima de estas cifras. Y qué decir si la exigencia de accesibilidad plena exige, además, la instalación de un ascensor en los edificios carentes del mismo o, habiéndolo, cualquier otra intervención para alcanzar la cota o, etc. Se recuerda que la fecha para la realización de ajustes razonables en materia de accesibilidad en edificios existentes está establecida para finales de 2017.

Y surgen los primeros problemas. Algunos de los técnicos intervinientes en la redacción de IEEs y/o proyectos de rehabilitación entrevistados afirman haber sido presionados en alguna ocasión por parte de las comunidades de propietarios para que los problemas y carencias que pueda presentar el edificio sean minimizados, en lo posible, a la hora de realizar su valoración. En otros casos, confiesan haber podido aprovechar los resquicios de la ley para reducir, por ejemplo, el ámbito de aplicación de la intervención, programando los trabajos por fases, con objeto de no superar el $25 \%$ de la superficie de la envolvente exigida por la norma en cada una de ellas o, alternativamente, planteándolos como simples trabajos de reparación puntual de lesiones.

\section{CONCLUSIONES}

Muchos de los edificios que conforman nuestro parque residencial van a requerir intervenciones integrales para subsanar las importantes lesiones y carencias que presentan. Especialmente, los edificios de vivienda colectiva construidos durante el periodo desarrollista, desde principios de los sesenta hasta mediados de los setenta, cuya débil y precaria envolvente exterior va a acaparar, a buen seguro, la mayor parte de las actuaciones futuras. Sin olvidar la necesidad añadida de muchos de ellos de tener que afrontar obras tendentes a mejorar la accesibilidad de forma obligatoria a partir de finales de 2017.

Debido a la exigencia de mejora de la eficiencia energética de la envolvente impuesta tras los cambios normativos de 2013, muchos edificios han comenzado a lucir nuevas pieles. En el caso de las fachadas, parte fundamental de la envolvente térmica, las soluciones con revestimiento continuo tipo SATE y las fachadas ventiladas con múltiples acabados parecen imponerse. $\mathrm{Y}$ tal y como se desprende del estudio realizado e independientemente de los diferentes condicionantes que influyen en cada caso, el coste medio por propietario de una rehabilitación de fachadas se puede establecer entre los diez mil y los veinte mil euros. Cantidades muy importantes si tenemos en cuenta, además, la importante pérdida del poder adquisitivo de los hogares desde el inicio de la crisis económica, pasando, según datos del Instituto Nacional de Estadística, de una renta de $30.045 €$ en el año 2009 hasta una renta de 26.730 $€$ en el año 2016, lo que supone una caída porcentual del $12 \%$. Considerando ambos parámetros, el incremento de coste generado por los cambios normativos y la pérdida de renta de los hogares, los datos indican que el esfuerzo económico medio para aquellos propietarios que tienen que hacer frente a esas obras se incrementa cerca de un $50 \%$ con respecto a la situación anterior, siendo especialmente relevante su repercusión en perfiles económicos de poder adquisitivo medios-bajos o bajos, mayoritarios en los edificios con mayor necesidad de renovación y con mayor dificultad para acceder a financiación privada por parte de las entidades de crédito.

La necesidad de mejorar las prestaciones de la envolvente aprovechando la ejecución de obras de reparación de lesiones viene dada desde instancias europeas y la $\mathrm{L}_{3} \mathrm{R}$ no hace más que trasponerla a la legislación española. No obstante, la propia ley deja bien a las claras que ante la fuerte crisis 
inmobiliaria que ha afectado principalmente a la obra de nueva planta, se pretende redirigir al castigado sector de la construcción hacia el mundo de la rehabilitación, generando la lógica duda de si las exigentes condiciones dispuestas en la Ley eran realmente tan necesarias. Y aunque las estrategias macroeconómicas y macroambientales, tanto europeas como estatales, pueden aconsejar elevar considerablemente el listón de las exigencias sobre las bases de una teórica futura amortización en el ahorro energético, la aprobación de ambiciosos planes de financiación pública para subvencionar las actuaciones previstas o la recuperación de un sector clave en la economía nacional como es el de la construcción, llegados a este punto, surge otra pregunta: Y... ¿̇quién paga todo esto? Según el análisis reflejado en un informe de WWF, el capital de inversión para la reforma de edificios en Europa puede provenir únicamente de seis fuentes: del gobierno, del propietario del edificio, del inquilino, del banco, del contratista de la reforma o del suministrador de energía (17). Pero, a día de hoy, la realidad es que cuando una comunidad de vecinos se ve obligada a afrontar obras de esta envergadura lo primero que ha de hacer es abonar su coste íntegro, sin garantía alguna de que pueda recibir ayudas económicas en el futuro. La prueba fehaciente de la incertidumbre e indefinición temporal a la que están sujetos los programas de ayuda es que durante un año y medio, entre mayo de 2016 y diciembre de 2017, no ha habido convocatoria alguna de ayudas públicas a la rehabilitación energética en edificios residenciales en el ámbito estatal, no cumpliéndose, por lo tanto, lo reflejado en el Preámbulo de la $\mathrm{L}_{3} \mathrm{R}$ cuando se dice que la Administración General del Estado "asegura la colaboración y la cooperación económica". Y en un momento como el actual, con altos niveles de paro e importantes restricciones a la financiación privada, basta con que uno o varios vecinos no puedan afrontar los pagos de obras de semejante envergadura para que las mejores intenciones rehabilitadoras se vengan abajo sin que por ello se reduzca ni un ápice, no lo olvidemos, el deber de conservación y la responsabilidad última de la comunidad de propietarios sobre el estado del inmueble y las consecuencias que de él se deriven.

La rehabilitación energética del parque residencial es necesaria y no se pone en cuestión. Faltaría más. Pero resulta evidente que el esfuerzo económico que va a requerir a gran parte de la ciudadanía en los próximos años debe ir acompañado no solo de compromisos y buenas intenciones sino de hechos y de políticas de ayudas públicas reales y de cuantía proporcional al coste exigido para que el proceso de modernización de nuestros edificios sea, nunca mejor dicho, sostenible y no se convierta en un problema social. Un permanente e interconectado chequeo por parte de las administraciones públicas de la efectividad de las diferentes medidas planteadas en el ámbito europeo para las ayudas a la rehabilitación (ayudas públicas directas, créditos y préstamos a bajo interés, implicación de los sectores financieros y de la construcción, etc), debería constituir la base de la elección e implementación de la mejor opción futura.

\section{REFERENCIAS}

(1) Etxepare, L.; Lizundia, I.; Sagarna, M.; Uranga, E.J.; Malles, E.; Ibarloza, E.; Ibarloza, A. (2015-17). Estudio del impacto en el coste, debido a las nuevas exigencias normativas, de la rehabilitación de la vivienda colectiva construida entre 1960 y 1980 en el País Vasco y sus consecuencias económicas y sociales. Proyecto de Investigación EHU14/56. UPV/EHU.

(2) Jefatura del Estado (2013). Ley 8/2013, de 26 de junio, de rehabilitación, regeneración y renovación urbanas. Boletín Oficial del Estado, $\mathrm{n}^{\mathrm{0}} 153$. España.

(3) Ministerio de Fomento (2013). Orden FOM/1635/2013, de 10 de septiembre, por la que se actualiza el Documento Básico DB-HE «Ahorro de Energía», del Código Técnico de la Edificación, aprobado por Real Decreto 314/2006, de 17 de marzo. Boletín Oficial del Estado, $\mathrm{n}^{0} 219$. España.

(4) Cuchí, A. Sweatman, P. (2011). Una visión-país para el sector de la edificación en España. Grupo de Trabajo sobre Rehabilitación (GTR).

(5) INE (2011). Censo de Población y Viviendas 2011. Edificios. Instituto Nacional de Estadística (INE)

(6) Capdevila, I., Linares, E., Folch, R. (2012). Eficiencia energética en la rehabilitación de edificios. Barcelona: Fundación Gas Natural Fenosa, Consejo Superior de los Colegios de Arquitectos de España.

(7) Labandeira, X., Linares, P. (2011). Potencial económico de reducción de la demanda de energía en España. Vigo: Economics for Energy.

(8) World Wildlife Fundation (2010). Potencial de ahorro energético y de reducción de emisiones de $\mathrm{CO}_{2}$ del parque residencial existente en España en 2020. Madrid: WWF.

(9) Hernández, H. (2017). Evaluación económica de inversiones de eficiencia energética en viviendas. Building \& Management, Vol. 1 (2): 36-45.

(10) De la Cruz Mera, A. (2013). La Ley 8/2013, de 26 de junio, de Rehabilitación, Regeneración y Renovación Urbanas. Revista digital CEMCI, 20, 1-21.

(11) CEOE. (2014). La rehabilitación de edificios como motor de crecimiento y empleo. Confederación Española de Organizaciones Empresariales, Comisión de infraestructuras y Urbanismo

(12) Ministerio de Sanidad, Servicios Sociales e Igualdad (2013). Real Decreto Legislativo 1/2013, de 29 de noviembre, por el que se aprueba el Texto Refundido de la Ley General de derechos de las personas con discapacidad y de su inclusión social. Boletín Oficial del Estado, ${ }^{0} 289$. España.

(13) Jefatura del Estado (1999). Ley 38/1999, de 5 de noviembre, de Ordenación de la Edificación. Boletín Oficial del Estado, $n^{0}$ 266. España.

(14) Commission to the European Parliament and the Council (2013). Financial support for energy efficiency in buildings. European Commission, COM(2013) 225 Final, Brussels.

(15) Ministerio de Energía, Turismo y Agenda Digital (2017). Resolución de 14 de diciembre de 2017, por la que se publica la Resolución de 6 de marzo de 2017, del Consejo de Administración, por la que se establecen las bases reguladoras se se- 
gunda convocatoria del programa de ayudas para actuaciones de rehabilitación energética de edificios existentes. Boletín Oficial del Estado, ${ }^{0}{ }^{309}$. España.

(16) Rubio de Val, J. (2015). Potencial del nuevo marco normativo para el impulso de la rehabilitación y la regeneración urbana en los ámbitos autonómico y local. Informes de la Construcción, 67(EXTRA-1): mo23, doi: http://dx.doi. org/10.3989/ic.14.072.

(17) World Wildlife Fundation (2012). Retos y oportunidades de financiación para la rehabilitación energética de viviendas en España. Madrid: WWF. 